\title{
Physical Contact and Loneliness: Being Touched Reduces Perceptions of Loneliness
}

\section{A. Heatley Tejada ${ }^{1}$ (D) R. I. M. Dunbar ${ }^{2}$ - M. Montero ${ }^{3}$}

Received: 23 March 2020 /Revised: 26 April 2020 / Accepted: 30 April 2020 / Published online: 26 May 2020

(C) Springer Nature Switzerland AG 2020

\section{Abstract}

Objective This article addresses the connection between loneliness and physical contact. Evolutionary and psychological research has shown that touch is an important part of bond-building and emotion communication; loneliness is intimately related to these elements as well. In this paper, we ask whether physical contact reduces feelings of loneliness — which might derive from evolutionary ancient bonding mechanismsdespite a cultural context that is relatively non-tactile.

Method An experimental study (40 participants, 13 males) tested for observable effects of touch on loneliness scores in a low-contact culture to analyse whether they respond positively to that stimulus despite cultural training against it.

Results Participants exposed to physical contact reported significantly lower neglect scores from their close relationships in a short loneliness scale, thus suggesting that there is an underlying mechanism that persists despite enculturation. The effects were particularly strong among single people, which could mean that lower loneliness among married people might be partly explained by the regular availability of physical contact. Participants in the experimental condition also showed a faster reduction in heart rate, interpreted as a sign of physiological wellbeing.

Conclusions These findings help to specify mechanisms within the evolutionary theoretical framework of loneliness that link internal feelings to environmental cues. This article aims at contributing to a more complex discussion on the interactions between emotions, cultural practices and psychological well-being.

Keywords Loneliness $\cdot$ Physical contact $\cdot$ Evolutionary psychology $\cdot$ Culture $\cdot$ Social distance

\section{A. Heatley Tejada ana.heatley@ciss-bienestar.org}




\section{Introduction}

Loneliness is related to a person's social bonds or the quality of an individual's social network (Hawkley et al. 2008). Human social relationships, particularly the closest most intimate ones, have a very tactile side to them (Cascio et al. 2018; Dunbar 2010). However, the link between social touch and loneliness has seldom been addressed. In scientific research, proxemics studies (Hall 1969; Sorokowska et al. 2017; Sussman and Rosenfeld 1982) measured physical contact in different cultures rather than the social significance and the emotional implications of tactile behaviour, which inform and shape the actual practice. Suvilehto et al. $(2015,2019)$ reported that, despite broad overall similarities, there are significant differences in the patterns of permissible bodily touching across cultures. Recently, neuro-psychological approaches to social touch have made significant progress in our understanding of the underlying mechanisms and social implications of human touch behaviour (Cascio et al. 2018; Su and Su 2018). Analysing the effects of social touch on loneliness is relevant to elucidate the evolutionary, cultural, and psychological processes that come into play in this relationship.

There are at least two reasons why analysing the effects of social touch on loneliness is important. Firstly, social touch is a powerful bonding mechanism that humans share with other primates that could constitute one environmental trigger for loneliness under an evolutionary perspective and one mechanism we use to reconnect with our social network. Secondly, it is important to place the analysis of the evolutionary mechanism of loneliness in real-life cultural contexts, given that tactile behaviour - and social interaction in general - is always culturally shaped.

The evolutionary theory of loneliness (Cacioppo et al. 2006, 2014) posits that feeling lonely is an alarm against survival risks associated with social isolation. The rationale is that loneliness arises with relationship quality decay and motivates us to invest time and effort to restore social bonds. Although relationship quality has been identified as the signal that triggers the loneliness alarm (Hawkley et al. 2008; Hawkley and Cacioppo 2010), it is not yet clear exactly what features of social interaction humans monitor to establish the quality of their relationships or what are the underlying mechanisms that activate and deactivate the alarm. Evolutionary features respond to specific processes but the quality of a relationship —or the perception of the quality - is a general abstraction of a collection of events and perceptions rather than a straight-forward unitary trait. In other words, to provide adaptive fitness, loneliness needs to be connected in some way to the (social) environment and not just to a subjective evaluation (satisfaction) or perception of the social network and/or social interaction. Thus, more detailed analysis is needed to understand how the internal mechanism of feeling lonely is connected to the external environment, and previous research suggests that physical contact might be involved in that connection.

Bonding in primates relies on tactile social activities (such as halo-grooming) and humans evolved to incorporate additional more time-efficient mechanisms such as singing, dancing and storytelling (Dunbar 2012). But modern humans still use and respond to physical bonding mechanisms (Dunbar 2010; Nummenmaa et al. 2016); affectionate touch has been found to have positive effects on relational well-being and it is associated with emotional depth in the relationship (Jakubiak and Feeney 2017; Suvilehto et al. 2015, 2019). Therefore, social touch might be one specific signal of the 
quality or depth of a relationship that could be involved in triggering loneliness as a warning against social disconnection.

As mentioned before, tactile behaviour varies widely among cultural groups and differences in the amount of touch, the forms of touch and its meanings or acceptability are not universal (Burleson et al. 2019). However, if touch is involved in an evolutionary mechanism, some of its effects must remain even in the face of contradicting cultural practices, given that evolutionary features are not culture-specific. Therefore, it is important to discriminate cultural practices from evolutionary processes - which is not easy. However, one strategy to address this difficulty is to analyse the effects of touch on loneliness in a cultural context that does not promote physical contact to distinguish cultural expectations as potential confounders.

The aim of the research reported in this paper is to examine the relationship between touch and loneliness. This topic is relevant because if loneliness is taken as an evolutionary feature, it needs to be connected to the environment, respond to it, and provide adaptive fit. But the specific environmental cues that trigger loneliness are not clearly identified yet in the evolutionary approach. Thus, we experimentally tested whether touch — as an evolutionarily ancient mechanism for social bonding - still affects loneliness despite contradicting social practices and values. We firstly address the theoretical argument that touch (or lack thereof) could act as an environmental cue for social isolation by discussing existing literature on the connection between physical contact and loneliness. Secondly, we present and discuss the evidence from an experimental study, which showed reduced levels of loneliness (neglect item) among people who received physical contact, compared to those who did not.

This research adds to the current evolutionary theory on loneliness by suggesting a novel research line on the specific mechanisms that trigger or deactivate the loneliness alarm (i.e., physical contact) with an approach that considers the specificities of the cultural context in which individuals actually live and interact (e.g., cultural expectations of independency and self-reliance).

\section{The Theory}

\section{Touch and Loneliness in Individualistic Low-Contact Societies}

Touch and physical displays of warmth or caring are important for human social bonding and psychological wellbeing (Field 2010; Gallace and Spence 2010; Jakubiak and Feeney 2017). There is evidence suggesting that touch, emotional depth or relationship quality, and loneliness are interconnected. Loneliness is related to poor relationship quality, particularly within the core of the social network (Heatley Tejada et al. 2017; Jackson et al. 2000), and those relationships, deep emotional ties, seem to rely heavily on physical contact and tactile ways of displaying affection (Dunbar 2010; Suvilehto et al. 2019). In addition, psychological research has shown that activities involving sensory perception - touch in particular - have therapeutic value for children who feel lonely (Bullock 1993; De Chomaso 1971). Although the pathways seem straightforward, the connection between these variables has seldom been directly addressed.

Although their role in human life is crucial, relationships and touch are both affected by cultural differences. Cultural norms regulate touch behaviour (Suvilehto et al. 2015, 
2019) and by doing so they could have an effect over bonding, the sense of connectedness, and loneliness feelings. Some "cold" or low-contact cultures associate independence and self-reliance with not needing physical contact, or rather they associate high contact needs with dependency or weakness. This is the case in Anglo-Saxon societies, which are considered low-contact and individualistic (Beaulieu 2004; Hofstede 2001; Sorokowska et al. 2017; Triandis 2001). In those individualistic settings, the ideal of independence and self-reliance might result in less social touch and, potentially, more loneliness. In fact, previous studies have found that individual level collectivism is associated with less loneliness (Heu et al. 2019).

Western lifestyle and contemporary urban life are associated with growing levels of loneliness and extended periods of people living alone, which increase vulnerability to loneliness (Twenge et al. 2019). According to The Mental Health Foundation's report, most of the risks of experiencing loneliness associated to contemporary Western lifestyle relate to the impossibility of —or limitations to - obtaining close and intimate physical contact and bonding experiences (Griffin 2010). Loneliness is an increasing concern in individualistic and low-contact societies (Cacioppo and Cacioppo 2018; Griffin 2010; Murthy 2017) ${ }^{1}$ even though people are used to reduced physical contact and most of them strive to become independent and self-reliant.

It thus seems that cultural training in low contact, self-reliance and independence does not "immunize" against feeling lonely. What needs to be further analysed is whether observable effects of touch over loneliness remain in the presence of cultural training in low-contact societies. To put the latter to test, we designed an experimental study where people from a low-contact individualistic society (UK) were exposed to physical contact with another person to examine if they reported lower loneliness scores than a control group. This would allow us to observe a) if touch has an observable effect over loneliness scores, and b) whether there is evidence of an underlying (potentially evolutionary) mechanism that persists despite (i.e. is not annulled by) a cultural context that discourages touch in usual social interaction.

\section{The Experiment}

\section{Method}

To analyse the effects of physical contact on loneliness in a low-contact culture we designed an experimental study that aimed to assess potential differences in loneliness scores after a brief exposure to physical contact (light rub on the back of the participants' hands).

Participants 42 volunteers were recruited in the UK and randomly assigned to two experimental groups (physical contact and control). Two participants had to be removed from the final sample due to inconsistent heart rate readings or not following given instructions. Final sample was formed by 40 participants ( 27 females, 13 males), aged 27 on average $(\mathrm{SD}=13.61)$, of which $22(55 \%)$ reported being in a romantic

\footnotetext{
${ }^{1}$ In 2017 there was a special commission on loneliness (Jo Cox Commission) that found worryingly high levels of loneliness among British people of all ages. In 2018, a minister for loneliness was appointed in a new role created specifically to address this problem.
} 
Table 1 Relationship status and gender distribution by experimental group

\begin{tabular}{lll}
\hline & In a relationship & Single \\
\hline Physical contact & 11 & 10 \\
& $(7$ females, 4 males $)$ & (7 females and 3 males $)$ \\
Control & 11 & 8 \\
Total & $(7$ females, 4 males $)$ & $(6$ females and 2 males $)$ \\
\hline
\end{tabular}

relationship. Relationship status and gender distribution by experimental groups is shown in Table 1.

Measures and Procedure Upon arrival, participants were informed of the activities involved in the experiment, requested to provide informed consent ${ }^{2}$ and wear a heart rate monitor throughout the length of the experiment. They were randomly assigned to the experimental condition (having a few drops of oil rubbed to the back of their hands by the researcher) or to a control group (rubbing the oil on themselves). After that, all participants completed a questionnaire containing social network profiling, attachment style and loneliness measures, and an emotion recognition task.

Heart rate (HR) was recorded with a Polar $\mathrm{H} 7$ monitor and results were processed using Kubios software. The aim of having HR recordings was to detect potential differences in unconscious physiological responses to the experimental condition. This was considered relevant, given that loneliness has been found to have significant effects over HR. ${ }^{3}$

Loneliness was measured with the Multi-faceted Loneliness Inventory (IMSOL) (Montero y López Lena 1999). This instrument was previously tested in a sample of 217 British participants. Reliability coefficient was $\alpha=.830$ and the correlation with the short version of the UCLA loneliness scale was $r=.8168, p<.001$ in that sample. Similar to the UCLA scale, the IMSOL measure consists of three questions on subjective feelings of loneliness (i.e., "How often do you feel neglected?", "How often do you feel lonely?", "How often do you feel emptiness in your life?") on a 1 (never) to 5 (always) frequency scale. A simple 1 (very little) to 10 (extremely) intensity scale was additionally included (i.e., "When you feel lonely, how intense would you say your loneliness feelings can be?") to explore potential differences in loneliness feelings' strength as opposed to frequency.

The "Reading the Mind in the Eyes" test (RMET) (Baron-Cohen et al. 2001) was also applied as part of the broader research project. This test measures emotion recognition abilities and consists of a series of 36 images showing the eyes area of the face only and participants are asked to choose the emotion that each image evokes from a list of four available options.

\footnotetext{
${ }^{2}$ This study was approved by University of Oxford Central University Research Ethics Committee and all participants gave informed consent before taking part in the experiment.

${ }^{3}$ Lower satisfaction with social support has been associated with higher heart rate reactivity after a stressful task (Nausheen et al. 2007), increased HR has been reported when participants face a stressful condition with no social support (Allen et al. 2002) and decreased cardiovascular reactivity has been found amongst the lonely (Cacioppo et al. 2000).
} 
Experimental Condition After obtaining informed consent, participants were asked to wear the heart rate monitor. Firstly, a 2.5 min test was taken as test reading in a sitting position with two aims: verifying that the HR monitor was working properly, and making sure that all participants' readings started from a resting position, allowing the effect of any previous physical activity on their heart rate (climbing the stairs to the experimental room, for instance) to fade out. For these reasons, this initial reading was not part of the analysis of HR results. Participants were able to see their on-line readings and the autonomic balance report as given by Elite HRV app ("morning readiness" test) at the end of this initial reading but they were not able to see their bpm or any other result during the rest of the experiment.

After this, participants received physical contact in the form of a light rub to the back of their hand. A female researcher (the same person in all cases) held the participant's hand and applied the oil with a light massage, lasting approximately one minute, until it was evenly distributed and absorbed. They were told that the oil was applied as part of the experiment to test if it enhanced autonomic balance during the task they were about to solve. The oil was simply baby oil and participants were debriefed on this at the end of the session. The researcher rubbed the oil to the hand of the participants randomly assigned to the experimental condition. Participants assigned to the control group were asked to rub the oil themselves.

Next, the participant was left alone to answer the questionnaire and task. The HR was recorded until they finished with all the questions. When they were finished, the HR recording was stopped, and participants were asked to remove the monitor. The session ended with debriefing and thanking them for taking part in the experiment.

Touch for apparently pragmatic reasons was selected for this study to avoid, firstly, making participants feel uncomfortable when experiencing physical contact from a complete stranger. And secondly, to avoid disclosing up front the purpose of the experimental condition, which would very likely bias participant's reaction to the stimulus and their responses to the questionnaire. The limitations of this strategy will be addressed in the "Discussion" section.

Analysis Analysis of Variance (ANOVA) was performed to examine the effects of the two conditions on loneliness scores and on heart rate. A strong artefact correction algorithm was applied ${ }^{4}$ to clean HR data before the analysis. The HR recording lasted for as long as it took the participant to answer all questions and tasks, and thus recording length varied form one participant to another. Therefore, to analyse comparable recordings, we only considered the first $20 \mathrm{~min}$ for the analysis, which was the time it took most participants to finish. The first half was divided into $2.5 \mathrm{~min}$ long periods to analyse in more detail the immediate reaction to the physical contact, given that the experimental condition occurred at the beginning of the session and we expected changes (if any) to occur during or immediately after the hand rub. Then, the overall average of the full $20 \mathrm{~min}$ of recording was calculated separately (see Table 4).

Descriptive statistics and loneliness, attachment and HR results can be found in Table 2.

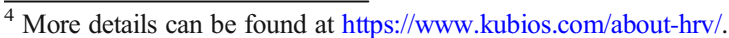


Table 2 Descriptive statistics and measurement results

\begin{tabular}{llllll}
\hline & $x$ & $\min$ & $\max$ & range & SD \\
\hline Age & 27.03 & 18 & 78 & 60 & 13.62 \\
Loneliness IMSOL & 6.2 & 3 & 10 & 7 & 1.81 \\
Loneliness intensity & 4.7 & 1 & 8 & 7 & 2.14 \\
Attachment anxiety & 2.74 & 1.33 & 4.33 & 3 & 0.67 \\
Attachment avoidance & 1.87 & 1 & 3.17 & 2.17 & 0.63 \\
HR & 72.20 & 41.50 & 110.2 & 68.67 & 13.83 \\
\hline
\end{tabular}

\section{Results}

We found significant differences between experimental and control groups (condition) and by relationship status in one of the loneliness scale items that reflects perceptions of neglect. Although there were no significant differences in the loneliness global average score, ANOVA showed that mean differences in the neglect item were significant between conditions $F(1,36)=5.034 p=.031$ and relationship status $F(1,36)=9.274$ $p=.004$. Post-hoc t-tests revealed that single participants that were exposed to physical contact reported significantly lower scores than single participants who did not receive physical contact $(p<.05)$ (see Fig. 1 below). Participants in a relationship had lower scores than single participants $(p<.05)$ and participants in the experimental condition had lower scores than the control group $(p<.1)$. Table 3 shows mean and standard deviation by experimental group and by relationship status.

Regarding heart rate analysis, participants in the physical contact condition showed a significant difference $(F(1,38)=3.759 p=.017)$ in heart rate mean in the overall average versus the first $2.5 \mathrm{~min}$ period. That is, pairwise comparisons showed that the average HR of people who received physical contact was significantly lower compared to the first reading of the experiment $(p<.05)$. There were no significant

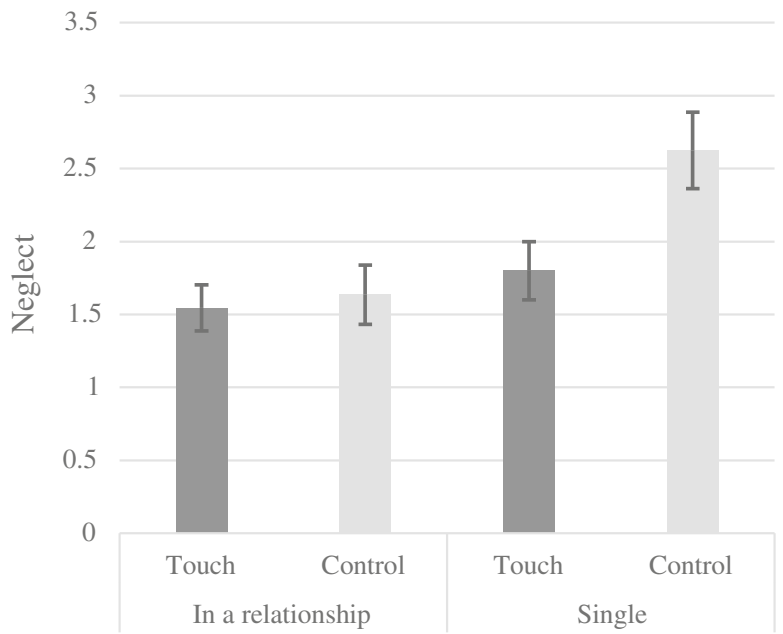

Fig. 1 Loneliness-neglect by experimental group and relationship status 
Table 3 Loneliness-neglect by experimental group and relationship status

\begin{tabular}{|c|c|c|c|c|c|c|}
\hline & \multicolumn{2}{|c|}{ In a relationship } & \multicolumn{2}{|c|}{ Single } & \multicolumn{2}{|c|}{ Overall } \\
\hline & $x$ & SD & $x$ & SD & $x$ & $\mathrm{SD}$ \\
\hline Physical contact & 1.55 & $(0.52)$ & 1.80 & $(0.63)$ & 1.67 & $(0.58)$ \\
\hline Control & 1.64 & $(0.67)$ & 2.63 & $(0.74)$ & 2.05 & $(0.85)$ \\
\hline Overall & 1.59 & $(0.59)$ & 2.17 & $(0.79)$ & 1.85 & $(0.74)$ \\
\hline
\end{tabular}

differences observed in the control group in any of the periods or the overall average. Table 4 gives mean beats per minute by experimental condition and by period, followed by the corresponding graph in Fig. 2.

The observed differences show that the physical contact condition is associated with a faster and more persistent reduction of HR. Decreasing beats per minute was expected given that the experiment was conducted in a sitting position and did not involve physical activity. However, participants who received physical contact had a steeper downward slope than the control group, which resulted in the average HR being significantly different (lower) than the first analysed reading in the experimental group.

\section{Discussion}

Experimental results suggest that physical contact has beneficial effects on loneliness and HR. Although the global loneliness score was not significantly different, the itemper-item analysis showed that people who received physical contact reported feeling significantly less neglected after receiving a small dose of touch, particularly among participants who were single (i.e., they declared not being currently dating or in a romantic relationship). Singles who received physical contact reported less neglect than singles who did not receive physical contact, lowering their scores to the levels reported by people who were in a relationship (see Fig. 1). This is consistent with previous studies that found that married people report less loneliness regardless of relationship quality (Beutel et al. 2017; Distel et al. 2010; Pinquart and Sörensen 2003). In this sense, our results seem to suggest that reduced loneliness in married people might relate

Table 4 Heart rate by experimental group and period

\begin{tabular}{|c|c|c|c|c|}
\hline \multirow[b]{2}{*}{ Period } & \multicolumn{2}{|c|}{ Physical contact } & \multicolumn{2}{|l|}{ Control } \\
\hline & $x$ & SD & $x$ & $\mathrm{SD}$ \\
\hline 1 & 70.60 & 9.67 & 73.27 & 22.90 \\
\hline 2 & 69.58 & 9.94 & 72.97 & 23.45 \\
\hline 3 & 68.55 & 10.58 & 72.11 & 22.58 \\
\hline 4 & 68.39 & 10.09 & 70.70 & 22.20 \\
\hline Overall & 68.14 & 9.30 & 71.46 & 21.35 \\
\hline
\end{tabular}

Note: Significant differences are highlighted 


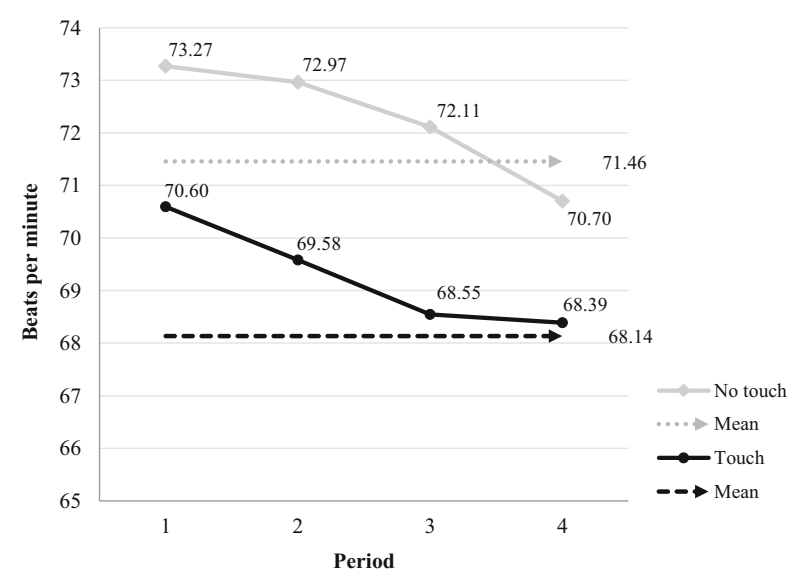

NOTE: The periods refer to the first 10 minutes from the start of the experiment itself (the experimental condition occurred during the first period). The mean refers to the average across the full $\mathbf{2 0}$ minutes of the experiment. We decided to decompose the first $\mathbf{1 0}$ minutes in smaller periods to analyse the immediate reaction to the experimental condition in more detail

Fig. 2 Heart rate behaviour by experimental group and period

to the availability of physical contact, independently from other aspects in the relationship, such as overall satisfaction or subjective perception of the quality of the relationship.

The observed effect of the experimental condition over heart rate suggests that physical contact has positive physiological effects, as other studies have also documented (Field 2010; Jakubiak and Feeney 2017; Lindgren et al. 2010). The fact that a small amount of physical contact is significantly associated with lower HR is a very promising result. We analysed the two groups (experimental and control) separately since our interest was on the trends in HR after receiving physical contact. This strategy allowed us to see that there is in fact a difference in behaviour which is independent of the initial differences in HR between the two groups. It is also an important indication of the positive effects touch has which is not susceptible to potential subjective biases in participant's responses to questionnaires.

The fact that the experiment was conducted in a low contact and individualistic society adds to the relevance of the results. This allowed us to minimise the potential influence of cultural practices of physical contact. We were able to ensure that people's reaction to touch is not due to cultural expectations, but rather is a direct positive reaction despite the fact that usual cultural expectations were not met. People culturally socialised to engage less in physical contact still respond to it. That is, in a cultural context where people are used to having less physical contact — with acquaintances and strangers in particular - receiving a small dose of touch has an observable effect. This suggests that even casual touch may play a more important role in mediating human interactions than we typically assume.

It could be argued that the results were due to the fact that, precisely because the experiment was conducted in a low-contact culture, participants reacted positively since they might feel touch-deprived in their daily lives and that people who get more physical contact on a daily basis would not react in the same way. Nonetheless, such 
an explanation still implies that people do not "get used" to lack of touch despite cultural training. This, in turn, implies that this is a human (not a cultural) need or response that is associated with perceptions of neglect and HR. Ruling out this alternative explanation would require replicating the experiment in a high contact cultural setting, which is an exciting project for future studies.

The importance of touch as a potential environmental cue for the loneliness alarm relates to the fact that, for loneliness to be an evolutionary feature, it needs to be connected to the environment in some way. Previous research has studied the relationship between loneliness and several internal factors, ranging from personality traits (Beadle et al. 2012; Cheng and Furnham 2002), genetics (Boomsma et al. 2005; Goossens 2012; McGuire and Clifford 2000), and physiological and biochemical processes (Cacioppo et al. 2000; Eisenberger and Lieberman 2004; Hawkley and Cacioppo 2010; Nausheen et al. 2007). Nonetheless, there are still rather few data on the environmental cues to which those internal factors respond, other than objective social isolation, which does not fully account for loneliness variance (de Jong Gierveld and van Tilburg 2016; Holt-Lunstad et al. 2015). Under an evolutionary perspective, the link between loneliness and environment is crucial, and for that reason this investigation opens up a potentially fertile line of research.

Although the small sample means that these results should be treated with caution, they nonetheless suggest that it is worth exploring the effect of touch on preventing or reducing loneliness and that touch could potentially explain married people's comparatively lower loneliness scores. This is a promising research avenue in terms of strategies to diminish loneliness and its negative impact on psychological and physiological wellbeing. It is also relevant to conduct further research on this issue in the context of a global coronavirus pandemic which is enforcing "social" distancing (i.e. physical distance), which is likely to decrease psychological well-being in many ways, loneliness being one of them.

Notwithstanding the theoretical implications and reported findings, this research has limitations. One of them is that the physical contact participants experienced was brief and of a pragmatic nature with no explicit social content. Touch with apparent pragmatic purposes and no social content was specifically selected for this study to avoid making participants feel uncomfortable when touched by a complete stranger and to avoid disclosing up front the reason for the physical contact, which could bias participant's responses. However, it is also possible that this cautionary strategy diminished the potential effects under study. Thus, further research based on more complex experimental designs where participants receive more physical contact - perhaps in a more explicitly social condition - is required. It could be interesting to analyse the effects of different forms, intensity, and duration of social touch (e.g. socially acceptable contact on specific areas of the body, as indicated by the touch maps of Suvilehto et al. 2015, 2019). It is also crucial to associate touch behaviour with social meaning (i.e., displays of affection, comforting, soothing) in future experimental designs and/or take this difference into account to have a better understanding of the real-life effects of physical contact on feelings of loneliness. Building on the current findings, future research might provide more data to establish the full potential of physical contact over loneliness feelings.

A further limitation concerns the generalisations that can be made based on this study. We selected a low-contact, individualistic society for methodological reasons, 
i.e., conducting the experiment in a cultural setting which endorses and promotes behaviours opposed to the experimental condition. Although this allowed us to separate cultural effects from experimental effects, further studies are needed before arriving at general conclusions about cross-cultural effects of physical contact on loneliness. Accounting for cultural effects — and estimating its magnitude — would require collecting data in different cultural groups. However, we were able to show that physical contact seems to reduce loneliness scores even among people who are culturally expected to be emotionally self-reliant and used to low physical contact in their daily lives. That is, the expected effects were observed in the most unlikely scenario, which indicates that it is worth exploring the issue further with a crosscultural perspective.

Future studies could also explore other aspects of this topic that might yield interesting insights. From a methodological perspective, it would be relevant to analyse whether lack of physical contact precludes loneliness or physical contact buffers loneliness, or both. This would help to clarify the causal pathways in the relationship and to establish the potential of touch in either preventing or alleviating loneliness. Also, more research on touch and loneliness could help expand our understanding of the evolutionary history of loneliness as an adaptive mechanism and could help us detail specific triggers for the loneliness alarm and its operational mechanisms, which currently are not clearly outlined. Also, it could help to better understand how emotional issues are resolved in the actual culturally-meaningful reality, which requires a more complex qualitative analysis.

Additionally, a comparative analysis of the differences in loneliness incidence, mechanisms and coping strategies in different cultures would be desirable. Culture has often been treated as a control variable, but the evidence reviewed here highlights the importance of looking deeper into the way in which cultural practices interact with emotion in general, and loneliness in particular. Human behaviour is always a psychological phenomenon, but it is also a cultural phenomenon too. Involving both dimensions in scientific research simultaneously is not only possible but necessary if the aim is to understand human social behaviour in all its complexity. Thus, this paper brings forward the need for placing the analysis of loneliness and other subjective well-being phenomena in a richer cultural context. We know that, to a certain extent, culturallydetermined lifestyles hinder or promote mental health, and mental health is becoming a growing concern in different public policy agendas. It is important to redefine the scientific approach to the interaction between culture and phenomena like loneliness and consider the implications for public and social policy.

\section{Conclusions}

This paper has examined the relationship between touch and loneliness within specific cultural settings, particularly individualistic and low-contact. The main contribution of the research reported here is to provide experimental evidence of a significant effect of physical contact on reduced loneliness perception, particularly among single people. Our results supported touch as a specific feature of the environment that could constitute a link between the internal emotional experience and external environmental factors or cues. To our knowledge, this is the first study to specifically test physical 
contact as an environmental trigger for loneliness in an explicitly evolutionary framework.

The fact that a small amount of physical contact produced observable effects is striking and suggests that the potential of this relationship/mechanism could be quite large. Also, if touch can reduce the perception of loneliness, this could imply that physical contact can alter people's perception of the quality of a relationship. Finally, our results point to some important psychological well-being implications in the context of a global pandemic that has enforced "social" (i.e. physical) distancing, where touch deprivation will expand and potentially affect more people.

Rising loneliness in different countries - whether due to increased awareness, to increasing incidence, or potentially to circumstantial events like a quarantine - highlights the need for developing efficient strategies to address the issue. Efficient designs require understanding the causal processes and mechanisms that underlie people's subjective conscious experience. They also require a good understanding of the way in which general mechanisms, which are theoretical abstractions, come into play with grounded cultural settings, which constitute the field of individual experiences. This research aimed to contribute to that endeavour by gaining theoretical insights without ignoring cultural background.

Acknowledgements A. Heatley would like to thank Dr. Luc Goosens for his valuable comments and useful discussions.

Funding Information A. Heatley's research was funded by CONACyT, supporting grant No. 369457.

R. Dunbar's research is supported by an ERC Advanced Investigator Award, grant No. 295663.

M. Montero's research is supported by UNAM-DGAPA-PAPIIT IN308816.

\section{Compliance with Ethical Standards}

Conflict of Interest On behalf of all authors, the corresponding author states that there is no conflict of interest.

Ethics Committee Approval and Informed Consent This study was approved by University of Oxford Central University Research Ethics Committee and all participants gave informed consent before taking part in the experiment.

\section{References}

Allen, K., Blascovich, J., \& Mendes, W. B. (2002). Cardiovascular reactivity and the presence of pets, friends, and spouses: The truth about cats and dogs. Psychosomatic Medicine, 739, 727-739. https://doi. org/10.1097/01.PSY.0000024236.11538.41.

Baron-Cohen, S., Wheelwright, S., Hill, J., Raste, Y., \& Plumb, I. (2001). The "Reading the mind in the eyes" test revised version: A study with normal adults, and adults with Asperger syndrome or high-functioning autism. Journal of Child Psychology and Psychiatry, and Allied Disciplines, 42(2), 241-251. https://doi. org/10.1111/1469-7610.00715.

Beadle, J. N., Brown, V., Keady, B., Tranel, D., \& Paradiso, S. (2012). Trait empathy as a predictor of individual differences in perceived loneliness. Psychological Reports, 110(1), 3-15. https://doi. org/10.2466/07.09.20.PR0.110.1.3-15.

Beaulieu, C. M. J. (2004). Intercultural study of personal space : A case study. Journal of Applied Social Psychology, 34, 794-805. https://doi.org/10.1111/j.1559-1816.2004.tb02571.x. 
Beutel, M. E., Klein, E. M., Brähler, E., Reiner, I., Jünger, C., Michal, M., Wiltink, J., Wild, P. S., Münzel, T., Lackner, K. J., \& Tibubos, A. N. (2017). Loneliness in the general population: Prevalence, determinants and relations to mental health. BMC Psychiatry, 17, 97. https://doi.org/10.1186/s12888-017-1262-x.

Boomsma, D. I., Willemsen, G., Dolan, C. V., Hawkley, L. C., \& Cacioppo, J. T. (2005). Genetic and environmental contributions to loneliness in adults: The Netherlands twin register study. Behavior Genetics, 35(6), 745-752. https://doi.org/10.1007/s10519-005-6040-8.

Bullock, J. R. (1993). Children's loneliness and their relationships with family and peers. Family Relations, 42, 46-49.

Burleson, M. H., Roberts, N. A., Coon, D. W., \& Soto, J. A. (2019). Perceived cultural acceptability and comfort with affectionate touch: Differences between Mexican Americans and European Americans. Journal of Social and Personal Relationships., 36, 1000-1022. https://doi.org/10.1177 /0265407517750005.

Cacioppo, J. T., \& Cacioppo, S. (2018). The growing problem of loneliness. The Lancet, 391, 426. https://doi. org/10.1016/S0140-6736(18)30142-9.

Cacioppo, J. T., Ernst, J. M., Burleson, M. H., McClintock, M. K., Malarkey, W. B., Hawkley, L. C., Kowalewski, R. B., Paulsen, A., Hobson, J. A., Hugdahl, K., Spiegel, D., \& Berntson, G. G. (2000). Lonely traits and concomitant physiological processes: The MacArthur social neuroscience studies. International Journal of Psychophysiology, 35(2-3), 143-154. https://doi.org/10.1016/S0167-8760(99 00049-5.

Cacioppo, J. T., Hawkley, L. C., Ernst, J. M., Burleson, M., Berntson, G. G., Nouriani, B., \& Spiegel, D. (2006). Loneliness within a nomological net: An evolutionary perspective. Journal of Research in Personality, 40(6), 1054-1085. https://doi.org/10.1016/j.jrp.2005.11.007.

Cacioppo, J. T., Cacioppo, S., \& Boomsma, D. I. (2014). Evolutionary mechanisms for loneliness. Cognition \& Emotion, 28(1), 3-21. https://doi.org/10.1080/02699931.2013.837379.

Cascio, C. J., Moore, D., \& McGlone, F. (2018). Social touch and human development. Developmental Cognitive Neuroscience, 35, 5-11. https://doi.org/10.1016/j.den.2018.04.009.

Cheng, H., \& Furnham, A. (2002). Personality, peer relations, and self-confidence as predictors of happiness and loneliness. Journal of Adolescence, 25(3), 327-339. https://doi.org/10.1006/jado.2002.0475.

De Chomaso, M. C. (1971). "Touch power" and the screen of loneliness. Perspectives in Psychiatric Care, 9(3), 112-118. https://doi.org/10.1111/j.1744-6163.1971.tb01082.x.

de Jong Gierveld, J., \& van Tilburg, T. G. (2016). Social isolation and loneliness. In Encyclopedia of Mental Health (pp. 175-178). https://doi.org/10.1016/B978-0-12-397045-9.00118-X.

Distel, M. A., Rebollo-Mesa, I., Abdellaoui, A., Derom, C. A., Willemsen, G., Cacioppo, J. T., \& Boomsma, D. I. (2010). Familial resemblance for loneliness. Behavior Genetics, 40(4), 480-494. https://doi. org/10.1007/s10519-010-9341-5.

Dunbar, R. I. M. (2010). The social role of touch in humans and primates: Behavioural function and neurobiological mechanisms. Neuroscience and Biobehavioral Reviews, 34(2), 260-268. https://doi. org/10.1016/j.neubiorev.2008.07.001.

Dunbar, R. I. M. (2012). Bridging the bonding gap: The transition from primates to humans. Philosophical Transactions of the Royal Society of London, 367B, 1837-1846.

Eisenberger, N. I., \& Lieberman, M. D. (2004). Why rejection hurts: A common neural alarm system for physical and social pain. Trends in Cognitive Sciences, 8(7), 294-300. https://doi.org/10.1016/j. tics.2004.05.010.

Field, T. (2010). Touch for socioemotional and physical well-being: A review. Developmental Review, 30(4), 367-383. https://doi.org/10.1016/j.dr.2011.01.001.

Gallace, A., \& Spence, C. (2010). The science of interpersonal touch: An overview. Neuroscience and Biobehavioral Reviews, 34(2), 246-259. https://doi.org/10.1016/j.neubiorev.2008.10.004.

Goossens, L. (2012). Genes, environments, and interactions as a new challenge for European developmental psychology: The sample case of adolescent loneliness. European Journal of Developmental Psychology, 9(4), 432-445. https://doi.org/10.1080/17405629.2012.673747.

Griffin, J. (2010). The lonely society? (p. 41). London: The Mental Health Foundation.

Hall, E. (1969). The hidden dimension : man's use of space in public and in private. In Anchor Books.

Hawkley, L. C., \& Cacioppo, J. T. (2010). Loneliness matters: A theoretical and empirical review of consequences and mechanisms. Annals of Behavioral Medicine, 40(2), 218-227. https://doi. org/10.1007/s12160-010-9210-8.

Hawkley, L. C., Hughes, M. E., Waite, L. J., Masi, C. M., Thisted, R. A., \& Cacioppo, J. T. (2008). From social structural factors to perceptions of relationship quality and loneliness: the Chicago health, aging, and social relations study. The Journals of Gerontology. Series B, Psychological Sciences and Social Sciences, 63(6), S375-S384. 
Heatley Tejada, A., Montero, M., \& Dunbar, R. (2017). Being unempathic will make your loved ones feel lonelier: Loneliness in an evolutionary perspective. Personality and Individual Differences, 116, 223232. https://doi.org/10.1016/j.paid.2017.04.042.

Heu, L. C., van Zomeren, M., \& Hansen, N. (2019). Lonely alone or lonely together? A cultural-psychological examination of individualism-collectivism and loneliness in five European countries. Personality and Social Psychology Bulletin, 45, 780-793. https://doi.org/10.1177/0146167218796793.

Hofstede, G. H. (2001). Culture's consequences. Comparing values, behaviors, institutions and organizations across nations. SAGE Publications. https://oi.org/10.1177/0022022110388567.

Holt-Lunstad, J., Smith, T. B., Baker, M., Harris, T., \& Stephenson, D. (2015). Loneliness and social isolation as risk factors for mortality: A meta-analytic review. Perspectives on Psychological Science, 10(2), 227237. https://doi.org/10.1177/1745691614568352.

Jackson, T., Soderlind, A., \& Weiss, K. (2000). Personality traits and quality of relationships as predictors of future loneliness among American college students. Social Behavior and Personality: An International Journal, 28, 463-470. https://doi.org/10.2224/sbp.2000.28.5.463.

Jakubiak, B. K., \& Feeney, B. C. (2017). Affectionate touch to promote relational, psychological, and physical well-being in adulthood: A theoretical model and review of the research. Personality and Social Psychology Review, 21, 228-252. https://doi.org/10.1177/1088868316650307.

Lindgren, L., Rundgren, S., Winsö, O., Lehtipalo, S., Wiklund, U., Karlsson, M., Stenlund, H., Jacobsson, C., \& Brulin, C. (2010). Physiological responses to touch massage in healthy volunteers. Autonomic Neuroscience: Basic and Clinical, 158(1-2), 105-110. https://doi.org/10.1016/j.autneu.2010.06.011.

McGuire, S., \& Clifford, J. (2000). Genetic and environmental contributions to loneliness in children. Psychological Science : A Journal of the American Psychological Society / APS, 11(6), 487-491. https://doi.org/10.1111/1467-9280.00293.

Montero y López Lena, M. (1999). Soledad: desarrollo y validación de un inventario multifacético para su medición. Universidad Nacional Autónoma de México.

Murthy, V. H. (2017). Work and the loneliness epidemic. Harvard Business Review.

Nausheen, B., Gidron, Y., Gregg, A., Tissarchondou, H. S., \& Peveler, R. (2007). Loneliness, social support and cardiovascular reactivity to laboratory stress. Stress (Amsterdam, Netherlands), 10(1), 37-44. https://doi.org/10.1080/10253890601135434.

Nummenmaa, L., Tuominen, L., Dunbar, R. I. M., Hirvonen, J., Manninen, S., Arponen, E., Machin, A., Hari, R., Jääskeläinen, I. P., \& Sams, M. (2016). Reinforcing social bonds by touching modulates endogenous $\mu$-opioid system activity in humans. Neurolmage, 138, 242-247.

Pinquart, M., \& Sörensen, S. (2003). Risk factors for loneliness in adulthood and old age: a meta-analysis. In S. Shohov \& S. P. Shohov (Eds.), Advances in psychology research (pp. 111-143). Hauppauge, NY: Nova Science Publishers.

Sorokowska, A., Sorokowski, P., Hilpert, P., Cantarero, K., Frackowiak, T., Ahmadi, K., Alghraibeh, A. M., Aryeetey, R., Bertoni, A., Bettache, K., Blumen, S., Błażejewska, M., Bortolini, T., Butovskaya, M., Castro, F. N., Cetinkaya, H., Cunha, D., David, D., David, O. A., .. Pierce, J. D. (2017). Preferred interpersonal distances: A global comparison. Journal of Cross-Cultural Psychology. https://doi. org/10.1177/0022022117698039.

$\mathrm{Su}$, J., \& Su, Y. (2018). A touch-scaffolded model of human prosociality. Neuroscience \& Biobehavioral Reviews, 92, 453-463. https://doi.org/10.1016/j.neubiorev.2018.07.008.

Sussman, N. M., \& Rosenfeld, H. M. (1982). Influence of culture, language, and sex on conversational distance. Journal of Personality and Social Psychology, 42(1), 66-74. https://doi.org/10.1037/00223514.42.1.66.

Suvilehto, J., Glerean, E., Dunbar, R. I. M., Hari, R., \& Nummenmaa, L. (2015). Topography of social touching depends on emotional bonds between humans. Proceedings of the National Academy of Sciences, USA, 112, 13811-13816.

Suvilehto, J. T., Nummenmaa, L., Harada, T., Dunbar, R. I. M., Hari, R., Turner, R., Sadato, N., \& Kitada, R. (2019). Cross-cultural similarity in relationshipspecific social touching. Proceedings of the Royal Society B: Biological Sciences., 286, 20190467. https://doi.org/10.1098/rspb.2019.0467.

Triandis, H. C. (2001). Individualism-collectivism and personality. Journal of Personality, 69(6), 907-924. https://doi.org/10.1111/1467-6494.696169.

Twenge, J. M., Spitzberg, B. H., \& Campbell, W. K. (2019). Less in-person social interaction with peers among US adolescents in the 21st century and links to loneliness. Journal of Social and Personal Relationships, 36(6), 1892-1913.

Publisher's Note Springer Nature remains neutral with regard to jurisdictional claims in published maps and institutional affiliations. 


\section{Affiliations}

\section{A. Heatley Tejada ${ }^{1}$ - R. I. M. Dunbar ${ }^{2} \cdot$ M. Montero ${ }^{3}$}

1 Inter-American Conference on Social Security, San Ramón s/n, Col. San Jerónimo Lídice, 10200 CDMX, Mexico

2 University of Oxford, New Radcliffe Building, Oxford OX2 6GG, UK

3 Universidad Nacional Autónoma de México, Facultad de Psicología, Ciudad Universitaria, 04510 CDMX, Mexico 